\title{
A Systematic Literature Review on Relationships Between Computational Thinking and Mathematics
}

Original Title: Uma Revisão Sistemática da Literatura Sobre as Relações Entre o Pensamento Computacional e a Matemática

\author{
Thiago Barcelos ${ }^{1,4}$, Roberto Muñoz ${ }^{2,3}$, Rodolfo Villarroel $^{3}$, Ismar Silveira ${ }^{4}$ \\ ${ }^{1}$ Instituto Federal de Educação, Ciência e Tecnologia de São Paulo - Guarulhos, SP - Brasil \\ ${ }^{2}$ Escuela de Ingeniería Civil Informática, Universidad de Valparaíso - Chile \\ ${ }^{3}$ Pontificia Universidad Católica de Valparaíso - Chile \\ ${ }^{4}$ Universidade Presbiteriana Mackenzie - São Paulo, SP - Brasil
}

\begin{tabular}{l}
\hline ARTICLE INFO \\
\hline Article history: \\
Received 16 June 2018 \\
Accepted 25 August 2018 \\
Available online July 1st 2018 \\
\hline Keywords: \\
Computational thinking \\
Mathematics \\
Systematic Literature Review \\
\hline
\end{tabular}

ISSN: 2595-9077

ABSTRACT

DOI: JCThink.2018.v2.n1.23

INTRODUCTION: A better understanding of the relationships between Computational Thinking and disciplines already present in the school curriculum may help the identification of possible educational benefits. This is particularly relevant in the case of Mathematics, which is a subject that constitutes itself as a hurdle to students in many Latin-American countries. OBJECTIVE: Identify and analyze didactic activities related to Computational Thinking and Mathematics reported in the literature regarding the target public, developed skills and contents, as well as research methods used to identify learning outcomes. METHODS: A Systematic Literature Review (SLR) was conducted, including studies published between 2006 and 2015 that included a description of didactic activities that developed Computational Thinking together with skills or contents related to Mathematics as well as an evaluation of learning outcomes. RESULTS: 59 studies were included in the revision. A wide variety of mathematical topics is being developed, with some emphasis on Algebra, Calculus and also higher-order thinking skills. Still $32.2 \%$ of included studies only present informal or anecdotal evidences of learning. CONCLUSION: In the last two years of the revision there was an increase in the number of activities focused on basic educational levels. Even though recently more rigorous methodological procedures were used to evaluate learning effects, still half of the studies with formal evaluation of learning outcomes use a single data source. Also, there are few studies focused on Math modelling and teacher training.

\section{RESUMO}

INTRODUÇ̃̃O: Uma melhor compreensão das relações entre o Pensamento Computacional e disciplinas já presentes no currículo da educação básica pode contribuir para a identificação de possíveis benefícios educacionais. Isso é particularmente relevante no caso da Matemática, que é uma disciplina que se constitui como um obstáculo para muitos estudantes em países latino-americanos. OBJETIVO: Identificar e analisar atividades didáticas relacionadas ao Pensamento Computacional e à Matemática, reportadas na literatura, em relação ao seu públicoalvo, competências e conteúdos desenvolvidos, e métodos de pesquisa utilizados para identificar resultados de aprendizagem. MÉTODO: Uma Revisão Sistemática da Literatura (RSL) foi realizada, incluindo estudos publicados entre 2006 e 2015 que incluíram uma descrição de atividades didáticas que desenvolveram o Pensamento Computacional conjuntamente com habilidades ou conteúdos matemáticos, bem como uma avaliação da aprendizagem. RESULTADOS: 59 estudos foram incluídos na revisão. Vários tópicos matemáticos são desenvolvidos, com predominância da Álgebra, Cálculo e habilidades cognitivas de alto nível. Ainda, 32,2\% dos estudos apresentam apenas evidências informais ou episódicas da aprendizagem. CONCLUSÃO: Nos últimos dois anos da revisão verifica-se um aumento do desenvolvimento de experiências na educação básica e um maior rigor metodológico na avaliação dos efeitos de aprendizagem. Por outro lado, cerca de metade dos estudos com avaliação formal da aprendizagem ainda utilizam uma única fonte de dados para análise. Ainda, se apresenta uma carência de estudos relacionados à Modelagem Matemática e à formação de professores.

\section{Introdução}

A comunidade de pesquisa em Informática na Educação tem considerado que a Ciência da Computação deveria fazer parte do currículo escolar desde as séries iniciais, sendo assim posicionada no mesmo nível das chamadas "ciências básicas", como a Física, a 
Biologia e a Química. No entanto, a motivação para essa proposta tem sido motivada pela Ciência da Computação em si, já que o principal incentivo para ensinar habilidades relacionadas à Computação tem sido promover a fluência digital entre os estudantes. Hood e Hood afirmam que a chave para obter a fluência dos estudantes em Tecnologia da Informação é incorporá-la ao currículo escolar (Hood \& Hood, 2005). Porém tal fluência não deveria ocorrer por intermédio do domínio de um conjunto de técnicas, mas sim por uma organização do pensamento voltada à resolução de problemas.

Por essa razão, um conjunto de competências e habilidades associadas à Ciência da Computação deveria ser desenvolvido pelos estudantes desde os primeiros anos da educação básica. Wing nomeou tal conjunto como Pensamento Computacional (Wing, 2006). Esse termo é atualmente utilizado para descrever os processos cognitivos relacionados à abstração e decomposição para permitir a resolução de problemas utilizando recursos computacionais e estratégias algorítmicas, dentre outras habilidades. A definição foi criticada por alguns autores por ser muito abrangente (Hu, 2011; Hemmendinger, 2010). No entanto, é possível identificar imediatamente algumas convergências entre essa definição e competências e habilidades relacionadas à Matemática. Polya, em seu clássico trabalho sobre a resolução de problemas matemáticos, apontou que a abstração (definida como a combinação de analogia, generalização e especialização) e a decomposição de problemas são cruciais para o sucesso na resolução de problemas (Polya, 2004).

Por outro lado, a Matemática se constitui como um obstáculo para os estudantes de vários países, em particular os latino-americanos. Conforme Aedo e Walker (2012), a pontuação média dos exames de Matemática do PISA na Argentina, Brasil, Chile, México e Peru é aproximadamente 100 pontos inferior à pontuação média obtida nos outros países que compõem a OECD (Organization for Economic Co-operation Development). Isso equivale a uma defasagem de cerca de dois anos na educação dos estudantes dos cinco países citados anteriormente.

Para incorporar atividades de desenvolvimento do Pensamento Computacional na educação básica é necessário considerar qual seria o impacto de tais atividades na aprendizagem dos conteúdos escolares tradicionais. Isso é particularmente importante por ser o ensino básico, em vários países, um ambiente no qual várias prioridades, ideologias e filosofias lutam por atenção (CSTA, 2011). Relações entre a Matemática e a Ciência da Computação no ambiente educacional já foram discutidas anteriormente (Ke, 2014; Krone, Sitaraman, \& Hallstrom, 2011). Dessa forma, é razoável inferir que o processo de ensino-aprendizagem de Matemática seria beneficiado de alguma forma pela incorporação do Pensamento Computacional ao currículo do ensino básico.

Muitas atividades visando o desenvolvimento do Pensamento Computacional associado a competências, habilidades e conteúdos matemáticos foram reportadas na literatura nos últimos anos, o que motiva uma análise detalhada dos resultados educacionais obtidos por intermédio dessas iniciativas. Dessa forma, este artigo apresenta uma Revisão Sistemática da Literatura com o objetivo de identificar como as relações entre a Matemática e o Pensamento Computacional foram demonstradas por meio do desenvolvimento de atividades didáticas descritas na literatura. A revisão foi guiada pelas seguintes perguntas de pesquisa:

(Q1) Como atividades didáticas relacionadas ao Pensamento Computacional e à Matemática foram desenvolvidas e qual seu público-alvo?

(Q2) Que competências, habilidades e conteúdos do Pensamento Computacional e da Matemática são ensinados nas atividades didáticas?

(Q3) Que métodos e técnicas de pesquisa são empregados para identificar possíveis efeitos de aprendizagem? 


\section{Métodos}

A revisão sistemática foi desenvolvida conforme as diretrizes propostas por Kitchenham (2004) e posteriormente sistematizadas por Wohlin et al. (2012), enumeradas a seguir:

Estágio 1. Planejamento da revisão, envolvendo: (1.1) Identificação da necessidade de uma revisão; (1.2) Definição da pergunta de pesquisa e (1.3) Definição do protocolo da revisão.

Estágio 2. Condução da revisão, envolvendo: (2.1) Identificação das pesquisas, (2.2) Seleção de estudos primários, (2.3) Verificação da qualidade dos estudos, (2.4). Extração e monitoramento de dados e (2.5) Síntese de dados.

Estágio 3. Relato dos resultados.

\subsection{Planejamento da revisão}

Inicialmente, uma pesquisa preliminar foi realizada em três repositórios online (ACM, IEEE e Google Acadêmico), a partir da qual não foi encontrada uma revisão da literatura com o mesmo objetivo da proposta deste trabalho. Assim, esta pesquisa foi útil para indicar a necessidade de uma revisão (Atividade 1.1). As questões de pesquisa para a revisão (Atividade 1.2) e as suas motivações já foram descritas na Seção 1 deste artigo.

O passo seguinte está relacionado com a definição do protocolo da revisão (Atividade 1.3). Segundo Wohlin et al. (2012) o protocolo deve indicar a estratégia de pesquisa, que inclui os repositórios de pesquisa dos quais serão extraídos os estudos, definição de strings de busca e critérios de inclusão e exclusão. Cinco repositórios foram incluídos nesta revisão: ACM, IEEE Xplore, ERIC, ScienceDirect e SpringerLink. A pesquisa sobre as características e atividades do Pensamento Computacional é razoavelmente recente e, por isso, optamos por usar uma string de busca abrangente: "computational thinking" and ("math" or "mathematics"). O objetivo foi identificar a maior quantidade de estudos possível, disponíveis em língua inglesa, mesmo à custa de uma seleção manual mais trabalhosa.

Foram definidos dois critérios de inclusão. Para ser incluído na revisão, o estudo deveria indicar alguma relação entre o Pensamento Computacional e alguma competência, habilidade ou tópico relacionado à Matemática (critério CI1). Ainda, para ser incluído na revisão um estudo deveria descrever uma atividade didática e apresentar os resultados da sua avaliação (critério CI2). Como foi feita a opção por uma estratégia de busca inclusiva, foram incluídos tanto estudos que apresentaram resultados de experimentos controlados quanto estudos que apresentavam apenas evidências empíricas. Essa estratégia foi adotada para identificar quão precisa foi a identificação dos resultados educacionais. Da mesma forma, foram excluídos estudos que atendiam a algum dos critérios de exclusão abaixo:

(CE1) O artigo descreve um tutorial, demonstração, proposta de painel de discussão ou entrevista;

(CE2) Pensamento Computacional (ou o ensino de Computação) é o tema do artigo, no entanto não são apresentadas relações com a Matemática;

(CE3) $\mathrm{O}$ artigo está fora de contexto e aborda outro tema de pesquisa.

\subsection{Condução da revisão}

As buscas foram executadas no dia 17 de agosto de 2015 nos cinco repositórios selecionados, quando 977 documentos foram obtidos. Os primeiros dois autores deste artigo foram responsáveis por classificar os artigos baseando-se no título e resumo. Quando após este procedimento ainda não era claro se o artigo poderia ser incluído na revisão, ele era marcado para posterior revisão do seu texto completo. Ou seja, após a 
primeira fase da análise, os artigos eram classificados como incluído (I), excluído (E) ou marcado para verificação (V). Na segunda fase, o texto completo dos artigos do último grupo foi analisado, e a classificação final de tais artigos foi: incluído após verificação (IV) ou excluído após verificação (EV). As estatísticas de inclusão e exclusão após as duas fases da análise são apresentadas na Tabela 1.

\begin{tabular}{|l|c|c|c|c|c|}
\hline & \multicolumn{2}{|c|}{ Artigos incluídos } & \multicolumn{2}{c|}{ Artigos excluídos } & \multirow{2}{*}{ Total } \\
\hline Base & I & IV & E & EV & \\
\hline ACM & 33 & 8 & 490 & 27 & 558 \\
\hline ERIC & 4 & 0 & 10 & 0 & 14 \\
\hline IEEExplore & 10 & 12 & 116 & 17 & 155 \\
\hline ScienceDirect & 6 & 5 & 36 & 10 & 57 \\
\hline SpringerLink & 12 & 2 & 168 & 11 & 193 \\
\hline Total & 92 & \multicolumn{2}{|c|}{885} & 977 \\
\hline
\end{tabular}

Tabela 1. Estatísticas dos artigos obtidos

Pode-se verificar que a maioria dos artigos obtidos foi excluída da revisão - 885 de 997 documentos, aproximadamente 90\% do total. Na Tabela 2 são indicados os motivos para a rejeição dos artigos, associados aos critérios de exclusão mencionados anteriormente. Pode-se notar que a maioria dos artigos rejeitados estava, de fato, associado ao Pensamento Computacional e mencionava as palavras "math" ou "mathematics" mesmo que nenhuma relação entre os conceitos fosse discutida (CE2). Ainda, muitos artigos foram rejeitados por mencionarem as palavras da string de busca mas estarem fora do contexto desta revisão (CE3).

\begin{tabular}{|l|c|c|c|c|}
\hline Base & CE1 & CE2 & CE3 & Total \\
\hline ACM & 71 & 340 & 106 & 517 \\
\hline ERIC & 0 & 6 & 4 & 10 \\
\hline IEEExplore & 0 & 67 & 66 & 133 \\
\hline ScienceDirect & 0 & 20 & 26 & 46 \\
\hline SpringerLink & 4 & 59 & 116 & 179 \\
\hline Total & $\mathbf{7 5}$ & $\mathbf{4 9 2}$ & $\mathbf{3 1 8}$ & $\mathbf{8 8 5}$ \\
\hline
\end{tabular}

Tabela 2. Razões para rejeição de artigos

Não foi necessário filtrar as buscas pelo ano de publicação pois foi assumido que as publicações que abordaram o Pensamento Computacional iriam aparecer após a publicação de Wing (2006) que introduziu esse termo. Para uma primeira análise do interesse da comunidade científica nesse tema de pesquisa, os 92 artigos incluídos na revisão foram separados em dois grupos: o primeiro grupo (EXP), com 59 artigos, incluiu os estudos que descreveram a aplicação de uma atividade didática acompanhada de uma avaliação experimental dos resultados de aprendizagem. O segundo grupo (DI) foi formado por 33 artigos nos quais os autores apresentavam discussões conceituais das relações entre o Pensamento Computacional e a Matemática com base em seu ponto de vista, análise documental ou outro tipo de estudo teórico. Também foram incluídos neste grupo os artigos que descreviam propostas de atividades didáticas sem nenhuma descrição de sua aplicação ou avaliação. 
Um gráfico apresentando o número de artigos publicados em ambos os grupos por ano de publicação (Figura 1) indica que há um interesse crescente da comunidade científica pela criação e avaliação de experiências didáticas. As primeiras três experiências didáticas relacionadas ao Pensamento Computacional e à Matemática foram reportadas em 2008, sendo que uma tendência crescente é verificada desde 2011, com uma pequena queda em 2014. No entanto, sete artigos descrevendo experimentos foram identificados apenas nos primeiros oito meses de 2015, o que pode indicar que ainda há grande interesse por esse tipo de trabalho. As primeiras discussões conceituais sobre o Pensamento Computacional foram identificadas em 2006, incluindo o trabalho pioneiro de Jeanette Wing. Desde então a quantidade de publicações desse tipo veio crescendo até 2011.

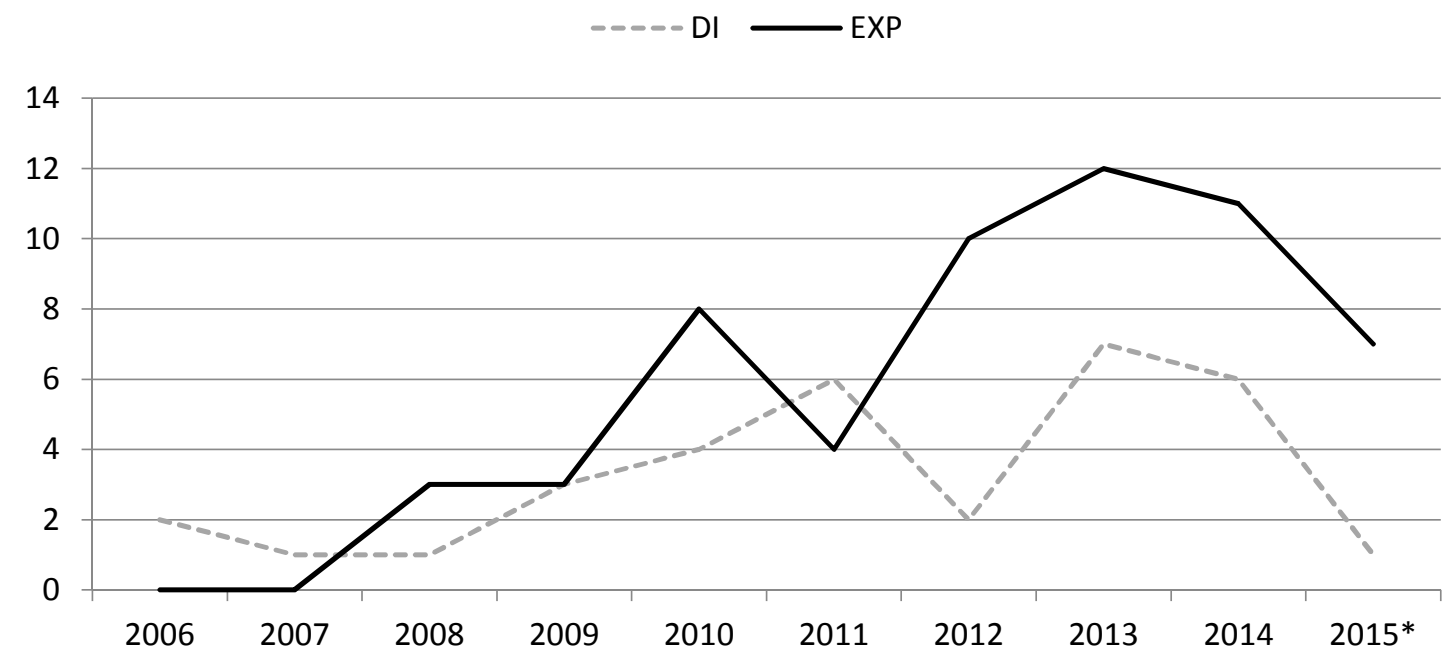

Figura 1. Artigos selecionados apresentando experiências didáticas (EXP) e discussões conceituais (DI) sobre o PC e a Matemática, por ano de publicação. Para o ano de 2015 os resultados são parciais.

Devido ao enfoque das perguntas de pesquisa propostas neste estudo, a partir deste ponto serão analisados e discutidos apenas os resultados obtidos com os artigos do grupo EXP.

\section{Resultados}

\subsection{Público-alvo}

Em cada artigo do grupo EXP foi identificado qual o público-alvo da atividade ou proposta relatada. Foi adotada na classificação a terminologia norte-americana para os níveis de ensino: elementary school, para os cinco primeiros anos da educação básica; middle school, para os três anos subsequentes da educação básica; e high school, para os quatro anos finais. Em geral, os autores de artigos que realizam experiências fora do contexto norte-americano também utilizaram essa terminologia para reportar suas experiências. Para os artigos em que essa estratégia não foi utilizada, o nível de ensino foi deduzido a partir da leitura de modo a uniformizar a contagem. Os dados são apresentados na Tabela 2.

\begin{tabular}{|l|c|c|l|c|c|}
\hline Nível de ensino & Quantidade & $\begin{array}{c}\text { \% artigos } \\
(\mathbf{n = 5 9 )}\end{array}$ & Públicos mistos & Quantidade & $\begin{array}{c}\text { \% artigos } \\
(\mathbf{n}=\mathbf{5 9})\end{array}$ \\
\hline Elementary School & 6 & $10,17 \%$ & $\begin{array}{l}\text { Elementary School / Middle } \\
\text { School }\end{array}$ & 2 & $3,40 \%$ \\
\hline Middle School & 14 & $23,73 \%$ & Middle School / High School & 3 & $5,08 \%$ \\
\hline
\end{tabular}




\begin{tabular}{|l|c|c|l|c|c|}
\hline High School & 8 & $13,56 \%$ & Middle School/Teachers & 1 & $1,69 \%$ \\
\hline Undergraduate students & 18 & $30,51 \%$ & $\begin{array}{l}\text { High School/ } \\
\text { Undergraduate students }\end{array}$ & 2 & $3,40 \%$ \\
\hline Teachers & 3 & $5,08 \%$ & $\begin{array}{l}\text { Undergraduate students / } \\
\text { Teachers }\end{array}$ & 1 & $1,69 \%$ \\
\hline \multicolumn{7}{|c|}{} & $\begin{array}{l}\text { Undergraduate students / } \\
\text { Middle School }\end{array}$ & 1 & $1,69 \%$ \\
\hline Total & $\mathbf{4 9}$ & $\mathbf{8 3 , 0 5 \%}$ & Total & $\mathbf{1 0}$ & $\mathbf{1 6 , 9 5 \%}$ \\
\hline
\end{tabular}

Tabela 3. Segmentação de estudos por nível de ensino do público-alvo.

É possível identificar que uma expressiva quantidade de estudos (33 artigos, ou $55,9 \%$ do total) apresentam experiências voltadas a um ou mais níveis da educação básica. Por outro lado, foram identificados 18 artigos que tem como alvo exclusivamente o ensino de graduação ou tiveram sua validação experimental realizada neste ambiente. Apesar da definição original de Pensamento Computacional (Wing, 2006) considerar o seu desenvolvimento como uma proposta voltada à educação básica, muitos pesquisadores vem também propondo atividades voltadas ao desenvolvimento do Pensamento Computacional de forma aliada à Matemática para alunos de graduação. Tais atividades são predominantemente voltadas para disciplinas introdutórias, visando atenuar os altos índices de evasão e reprovação em tais disciplinas. No entanto, também é necessário ressaltar que o interesse da comunidade em realizar experimentos na educação básica vem crescendo nos últimos anos: dos 33 artigos que relatam tais experiências, 16 (48,5\% do total) foram publicados entre os anos de 2013 e 2015.

\subsection{Software e materiais}

As experiências didáticas que desenvolvem a Matemática juntamente com o Pensamento Computacional vêm utilizando, predominantemente, ferramentas computacionais durante o seu desenvolvimento. A partir do mapeamento das ferramentas e materiais utilizados nos artigos, foram identificadas 58 ocorrências do uso de ferramentas computacionais e apenas 12 ocorrências do uso de atividades que não dependem do computador. Na Tabela 4 são apresentadas as dez ferramentas de software mais utilizadas e os materiais físicos utilizados, bem como as respectivas frequências de utilização. É preciso ressaltar que um estudo pode apresentar mais de uma ocorrência de uso de ferramentas de software e materiais.

\begin{tabular}{|l|c|}
\hline Software & Ocorrências \\
\hline Scratch & 9 \\
\hline Python & 5 \\
\hline Objetos de aprendizagem com finalidade específica & 6 \\
\hline Planilhas eletrônicas & 6 \\
\hline Java & 6 \\
\hline Kit de robótica & 4 \\
\hline NetLogo & 3 \\
\hline MATLAB / Octave & 3 \\
\hline Logo & 2 \\
\hline AgentCubes / AgentSheets & 2 \\
\hline Outros & 12 \\
\hline Total & $\mathbf{5 8}$ \\
\hline
\end{tabular}




\begin{tabular}{|l|c|}
\hline Material & Ocorrências \\
\hline Atividades CS Unplugged & 4 \\
\hline Atividades Lápis-e-papel & 8 \\
\hline Total & $\mathbf{1 2}$ \\
\hline
\end{tabular}

Tabela 4. Ferramentas de software e materiais didáticos utilizados.

Um variado conjunto de ferramentas computacionais é utilizado para o desenvolvimento de atividades. Das 58 ocorrências identificadas, pelo menos 34 referem-se a linguagens (ex: Java, MATLAB, Phyton), ambientes (ex: Scratch, AgentSheets) ou aplicações que permitem que o aluno desenvolva seus próprios artefatos computacionais. As atividades de "computação desplugada", que utilizam o conjunto de materiais CS Unplugged (Bell, Witten, Fellows, Adams, \& McKenzie, 2010), foram utilizadas na formação de professores (Jenkins, Jerkins, \& Stenger, 2012) e em um estudo de caso para avaliar a opinião de alunos sobre a associação entre a Matemática e a Ciência da Computação (Taub, Armoni, \& Ben-Ari, 2012).

\subsection{Estratégias para avaliação da aprendizagem}

O próximo passo foi analisar os artigos incluídos quanto ao rigor dos métodos e técnicas de pesquisa empregados na avaliação da aprendizagem. Dessa forma, foram definidos dois grupos: o primeiro contém os estudos que apresentam uma avaliação da aprendizagem formal, sob o ponto de vista do rigor da metodologia empregada. No caso de estudos que adotam o paradigma quantitativo, foram considerados como formais aqueles estudos que empregam um experimento, com atribuição aleatória de indivíduos em grupos de controle e experimental, ou quase-experimento, com a comparação entre grupos não-equivalentes, sem atribuição aleatória dos indivíduos a cada grupo (Trochim \& Donelly, 2006).

Para avaliar o rigor dos estudos que adotam o paradigma qualitativo foram utilizados dois dos critérios de validação propostos por Creswell e Miller (2000), denominados "engajamento prolongado em campo", entendido como o envolvimento do pesquisador no ambiente pesquisado por um período entre quatro meses e um ano, e a "descrição densa e rica" dos fenômenos observados, incluindo o ambiente e as falas dos sujeitos pesquisados. A avaliação foi feita de forma independente pelos dois primeiros autores deste artigo e eventuais discrepâncias na avaliação foram identificadas de forma conjunta a posteriori. Os resultados são apresentados na Tabela 5.

\begin{tabular}{|l|c|c|}
\hline Avaliação dos efeitos de aprendizagem & Quantidade & Percentual (n=59) \\
\hline $\begin{array}{l}\text { Formal } \\
\text { (experimento, quase-experimento ou estudo qualitativo rigoroso) }\end{array}$ & 40 & $67,80 \%$ \\
\hline $\begin{array}{l}\text { Informal } \\
\text { (estudo empírico ou observacional, ou amostra de tamanho insuficiente) }\end{array}$ & 19 & $32,20 \%$ \\
\hline Total & 59 & $100,00 \%$ \\
\hline
\end{tabular}

Tabela 5. Nível de rigor na avaliação dos efeitos de aprendizagem.

É possível identificar uma crescente tendência no uso de uma avaliação mais rigorosa dos efeitos de aprendizagem obtidos a partir de atividades didáticas envolvendo o Pensamento Computacional. Dos 40 artigos que apresentam avaliação formal, 18 (ou $45 \%$ do total) foram publicados entre 2013 e 2015, enquanto que no mesmo período foram identificados apenas 7 artigos com validação informal. O maior nível de rigor na avaliação também se refletiu na escolha de instrumentos de coleta e análise de dados 
para dar suporte aos estudos com avaliação formal, como é possível observar na Tabela 6. Cabe ressaltar que um mesmo estudo pode utilizar mais de um instrumento de coleta de dados.

A partir dos dados é possível identificar um uso intensivo de questionários pelos pesquisadores. Dentre os objetivos do uso desse instrumento, destacam-se a autoavaliação dos participantes sobre sua motivação e interesse sobre as atividades propostas, bem como o levantamento de perfil dos participantes. A avaliação da aprendizagem em si é tipicamente feita através de avaliações com conteúdo da Matemática escolar ou usando-se os resultados de avaliações oficiais já disponíveis. $\mathrm{O}$ uso conjunto de testes antes e após o oferecimento da experiência didática foi identificado em 15 estudos, enquanto que o uso de apenas um único teste após o oferecimento ocorreu em 9 estudos. Esse é outro indicativo da evolução do rigor metodológico ao longo do tempo, já que dos 15 estudos que utilizam pré e pós-testes, 9 foram publicados entre 2013 e 2015.

\begin{tabular}{|l|c|c|}
\hline Instrumento & Ocorrências & Percentual (n = 40) \\
\hline Questionário & 20 & $50.00 \%$ \\
\hline Pré e pós-teste & 15 & $37.50 \%$ \\
\hline Entrevista & 12 & $30.00 \%$ \\
\hline Apenas pós-teste & 9 & $22.50 \%$ \\
\hline Observação de aulas & 4 & $10.00 \%$ \\
\hline Análise de artefatos produzidos & 3 & $7.50 \%$ \\
\hline Taxa de aprovação & 2 & $5.00 \%$ \\
\hline Notas & 2 & $5.00 \%$ \\
\hline
\end{tabular}

Tabela 6. Instrumentos de coleta de dados utilizados em estudos com avaliação formal.

No entanto, como pode ser observado na Tabela 7, a maioria dos estudos com avaliação considerada como formal $(55 \%)$ utilizam um único instrumento de coleta de dados, o que pode ser uma limitação à validação final dos resultados, considerando o contexto educacional no qual a pesquisa está inserida. Esse aspecto será discutido na Seção 4.

\begin{tabular}{|c|c|c|}
\hline $\begin{array}{c}\text { Instrumentos de coleta } \\
\text { de dados }\end{array}$ & Quantidade & Percentual (n=40) \\
\hline 1 & 22 & $55.00 \%$ \\
\hline 2 & 10 & $25.00 \%$ \\
\hline 3 & 6 & $15.00 \%$ \\
\hline 4 & 2 & $5.00 \%$ \\
\hline
\end{tabular}

Tabela 7. Quantidade de instrumentos de coleta de dados utilizados nos estudos com validação formal.

\subsection{Competências, habilidades e conteúdos matemáticos}

A seguir foram analisadas as habilidades e conteúdos relacionados à Matemática que vem sendo desenvolvidos em conjunto com o Pensamento Computacional nos estudos realizados. A partir do agrupamento dos estudos que desenvolvem temas semelhantes foi possível identificar oito grupos de habilidades e conteúdos desenvolvidos:

- Habilidades cognitivas de alto nível: 18 estudos;

- Álgebra e Cálculo: 23 estudos;

- Álgebra Linear: 11 estudos 
- Geometria Planar: 16 estudos;

- Aritmética: 10 estudos;

- Física: 7 estudos;

- Estatística: 7 estudos;

- Modelagem Matemática: 5 estudos;

Novamente, deve-se observar que um mesmo estudo pode abordar habilidades ou conteúdos em mais de um grupo (por exemplo, Álgebra e Física). O primeiro grupo de estudos mencionado enfatiza o desenvolvimento de habilidades cognitivas mais gerais, discutindo suas relações com a Matemática. Dentre as habilidades desenvolvidas encontram-se a abstração e generalização para resolução de problemas (Jenkins et al., 2012), identificação de padrões (Boyce, Campbell, Pickford, Culler, \& Barnes, 2011) e a colaboração entre equipes por intermédio da linguagem matemática (Maxwell et al., 2013). Uma ampla variedade de conteúdos matemáticos vem sendo abordada, com alguma predominância para atividades que desenvolvem tópicos relacionados à Álgebra e ao Cálculo. Isso pode ser associado ao frequente uso de linguagens de programação e ambientes que induzem o uso de uma sintaxe baseada em conceitos algébricos como as variáveis. Além disso, de forma até esperada, os estudos que abordam o desenvolvimento de tópicos de Aritmética e Geometria Planar são predominantemente voltados ao público alvo da educação fundamental (elementary school), enquanto que os estudos que abordam tópicos de Álgebra e Cálculo, Estatística e Álgebra Linear são predominantemente voltados para alunos do ensino médio (high school) e de graduação.

\section{Discussão}

A partir da análise dos estudos incluídos nesta revisão é possível inferir que há um crescente interesse da comunidade científica em explorar as relações entre $o$ Pensamento Computacional e a Matemática. $\mathrm{O}$ aumento significativo, nos últimos anos, na quantidade de estudos nos quais atividades didáticas são apresentadas e testadas empiricamente contribui para essa conclusão.

Apesar de, originalmente, o conceito de Pensamento Computacional referir-se a habilidades que deveriam ser desenvolvidas por estudantes da educação básica, verificou-se que as atividades didáticas descritas não se restringem apenas a esse nível de ensino: cerca de $31 \%$ das atividades didáticas relatadas foram oferecidas a estudantes de graduação. Por um lado, isso indica que o desenvolvimento do Pensamento Computacional tem se mostrado útil na organização das pesquisas que tem como finalidade resolver os problemas de evasão e reprovação em cursos de graduação em Computação. Entretanto, isso não deixa de ser um "desvio" nos objetivos originais do Pensamento Computacional, já que o ensino de graduação é tipicamente o campo de aplicação mais próximo da realidade da pesquisa nas universidades. Algumas experiências didáticas apresentadas nos estudos têm como objetivo a formação de professores para disseminar o desenvolvimento do Pensamento Computacional; no entanto, tais iniciativas ainda são muito preliminares (apenas 5 estudos em um universo de 59) e necessitam de uma maior sistematização.

As atividades didáticas desenvolvidas se relacionam a uma ampla variedade de conteúdos matemáticos. A maioria das atividades faz uso de ferramentas computacionais, também bastante diversas. Esse é um indicativo da grande flexibilidade e potencial dos conceitos computacionais e das ferramentas de software como um suporte para ensinar e contextualizar a Matemática. Essa evidência contradiz parcialmente a revisão da literatura de Grover e Pea (2013), que menciona que o Pensamento Computacional não vinha sendo usado para ensinar outras disciplinas. 
O rigor nos métodos e técnicas de pesquisa utilizados para identificar possíveis ganhos de aprendizagem nos alunos também parece ter aumentado com o tempo, já que aproximadamente um terço dos artigos que apresentam uma validação mais rigorosa foram publicados nos últimos anos abrangidos pela revisão (2013 a 2015). De acordo com Husén (1997), o fenômeno da aprendizagem é inerentemente complexo e, dessa forma, seu estudo demanda um uso complementar dos paradigmas de pesquisa quantitativo e qualitativo. Uma consequência, do ponto de vista operacional, é a necessidade de coleta e análise de dados de diversas fontes de forma a obter uma compreensão mais precisa dos fenômenos observados. Nesse sentido, as limitações metodológicas identificadas por Grover e Pea (2013) também se verificam nos resultados desta revisão sistemática.

No grupo de estudos com uma validação mais rigorosa dos resultados, cerca de metade dos estudos não utiliza múltiplas fontes de dados para analisar os resultados do processo de ensino-aprendizagem por diferentes perspectivas. Destaca-se o uso intenso de questionários como instrumento de coleta de dados, geralmente para obter a autoavaliação dos estudantes sobre seu desempenho na atividade didática proposta ou sua aceitação em relação à atividade. Brennan e Resnick (2012) sugerem que as competências e habilidades adquiridas pelos estudantes são evidenciadas nos artefatos construídos por eles, uma teoria que também foi previamente evidenciada por outros autores (Basawapatna, Koh, Repenning, Webb, \& Marshall, 2011; Denner, Werner, \& Ortiz, 2012; Kafai, Franke, Ching, \& Shih, 1998). Apesar do fato da maioria das experiências didáticas empregarem ferramentas para criação de artefatos computacionais, somente três estudos utilizaram a análise dos artefatos criados, sejam eles digitais ou não.

\section{Conclusão}

O Pensamento Computacional representa um conjunto de competências e habilidades relacionadas à Ciência da Computação que deveriam ser desenvolvidas pelos estudantes da educação básica. No entanto, se mostra necessário compreender as relações do Pensamento Computacional com as disciplinas tradicionais do currículo escolar e quais os possíveis benefícios do desenvolvimento de estratégias didáticas conjuntas. Neste artigo apresentamos uma Revisão Sistemática da Literatura incluindo estudos publicados entre 2006 e 2015 que abordam as relações entre o Pensamento Computacional e a Matemática e cujos resultados permitem identificar os avanços e limitações da pesquisa nessa área.

A maioria das experiências didáticas descritas tem como público-alvo os alunos da educação básica, mas uma parcela relevante das experiências foi desenvolvida com alunos de graduação. Por outro lado, há relatos ainda insuficientes de experiências desenvolvidas na formação inicial e continuada de professores. Uma grande variedade de tópicos da Matemática vem sendo abordados, com alguma predominância para a Álgebra e o Cálculo. Vários estudos procuram desenvolver conjuntamente o Pensamento Computacional a Matemática por meio de habilidades de alto nível compartilhadas entre os dois paradigmas de pensamento, mas poucos estudos utilizam a construção e avaliação de modelos matemáticos e computacionais. Verifica-se um aumento do nível de rigor metodológico ao longo do tempo em relação aos métodos e técnicas de pesquisa empregados para avaliar efeitos de aprendizagem. Questionários e pré e pós-testes são os instrumentos de coleta mais utilizados, mas cerca de metade dos estudos utilizam uma única fonte de dados para avaliação.

É possível identificar um claro avanço na disponibilidade e variedade de atividades didáticas que envolvem o Pensamento Computacional e a Matemática. No 
entanto, ainda há públicos-alvo e habilidades matemáticas que vem sendo pouco exploradas pela comunidade. Por meio da identificação dessas limitações, esperamos que novos estudos venham a suprir essas lacunas. 


\section{Agradecimentos}

Roberto Muñoz é beneficiário da bolsa de Doutorado INF-PUCV 2015. Thiago Barcelos realizou parte deste trabalho com apoio de uma bolsa CAPES-PROSUP oferecida pela Universidade Cruzeiro do Sul. Os autores agradecem aos alunos Francisca Figueroa, Jorge Garin e Matias Camblor, da Universidade de Valparaíso, e Julio César de Ávila e Andreia Botto, do Instituto Federal de São Paulo, pelo apoio na obtenção e organização dos artigos analisados nesta pesquisa.

\section{Referências}

Aedo, C., \& Walker, I. (2012). Skills for the 21st Century in Latin America and the Caribbean. Washington, DC: The World Bank.

Basawapatna, A., Koh, K. H., Repenning, A., Webb, D. C., \& Marshall, K. S. (2011). Recognizing computational thinking patterns. In Proceedings of the 42nd ACM technical symposium on Computer science education (p. 245-250). New York: ACM.

Bell, T., Witten, I. H., Fellows, M., Adams, R., \& McKenzie, J. (2010). Computer Science Unplugged - An enrichment and extension programme for primary-aged children. Recuperado em 1 de agosto de 2013, de http://www.csunplugged.org

Boyce, A. K., Campbell, A., Pickford, S., Culler, D., \& Barnes, T. (2011). Experimental evaluation of BeadLoom game: how adding game elements to an educational tool improves motivation and learning. In Proceedings of the 16th annual joint conference on Innovation and technology in computer science education (p. 243-247). New York: ACM.

Brennan, K., \& Resnick, M. (2012). New frameworks for studying and assessing the development of computational thinking. In Proceedings of the 2012 annual meeting of the American Educational Research Association. Vancouver: American Educational Research Association.

Creswell, J. W., \& Miller, D. L. (2000). Determining validity in qualitative inquiry. Theory Into Practice, 39(3), 124-130.

Denner, J., Werner, L., \& Ortiz, E. (2012). Computer games created by middle school girls: Can they be used to measure understanding of computer science concepts? Computers \& Education, 58(1), 240-249.

Grover, S., \& Pea, R. (2013). Computational Thinking in K-12: A Review of the State of the Field. Educational Researcher, 42(1), 38-43.

Hemmendinger, D. (2010). A plea for modesty. ACM Inroads, 1(2), 4-7.

Hood, C. S., \& Hood, D. J. (2005). Toward integrating computing concepts into the K12 curriculum. SIGCSE Bulletin, 37(3), 375-375.

Hu, C. (2011). Computational thinking: what it might mean and what we might do about it. In Proceedings of the 16th annual joint conference on Innovation and technology in computer science education (p. 223-227). New York, NY, USA: ACM.

Husén, T. (1997). Research paradigms in education. In Educational research, methodology and measurement: an international handbook. Oxford: Pergamond.

Jenkins, J. T., Jerkins, J. A., \& Stenger, C. L. (2012). A plan for immediate immersion of computational thinking into the high school math classroom through a partnership 
with the Alabama math, science, and technology initiative. In Proceedings of the 50th Annual Southeast Regional Conference (p. 148-152). New York: ACM.

Kafai, Y. B., Franke, M. L., Ching, C. C., \& Shih, J. C. (1998). Game Design as an Interactive Learning Environment for Fostering Students' and Teachers' Mathematical Inquiry. International Journal of Computers for Mathematical Learning, 3(2), 149-184.

Ke, F. (2014). An implementation of design-based learning through creating educational computer games: A case study on mathematics learning during design and computing. Computers \& Education, 73(0), 26-39.

Kitchenham, B. (2004). Procedures for performing systematic reviews (Technical Report No. TR/SE-0401). UK: Keele University.

Krone, J., Sitaraman, M., \& Hallstrom, J. O. (2011). Mathematics throughout the CS curriculum. Jornal of Computing Sciences in Colleges, 27(1), 65-73.

Maxwell, A., Fogarty, R., Gibbings, P., Noble, K., Kist, A. A., \& Midgley, W. (2013). Robot RAL-ly international-Promoting STEM in elementary school across international boundaries using remote access technology. In 2013 10th International Conference on Remote Engineering and Virtual Instrumentation (REV) (p. 1-5). IEEE.

Polya, G. (2004). How to solve it: a new aspect of mathematical method. Princeton: Princeton University Press.

Taub, R., Armoni, M., \& Ben-Ari, M. (2012). CS Unplugged and Middle-School Students' Views, Attitudes, and Intentions Regarding CS. Trans. Comput. Educ., 12(2), $8: 1-8: 29$.

The CSTA Standards Task Force. (2011). CSTA K-12 Computer Science Standards. New York: ACM Computer Science Teachers Association.

Trochim, W. M. K., \& Donelly, J. P. (2006). Research methods knowledge base. Recuperado de http://www.socialresearchmethods.net/kb

Wing, J. M. (2006). Computational thinking. Communications of the ACM, 49(3), 3335.

Wohlin, C., Runeson, P., Höst, M., Ohlsson, M., Regnell, B., \& Wesslén, A. (2012). Systematic Literature Reviews. In Experimentation in Software Engineering (p. 45-54). Springer Berlin Heidelberg. 